\title{
Literatur- und Quellenverzeichnis
}

\author{
Angermann, A., \\ Crusen, W.G., \\ Schmidt, W. \\ Auernhammer, Herbert \\ Bayerischer Landtag \\ „Bayerisches Infor- \\ mationssystem"
}

Bereska, Detlev

Berger-Damiani, Erich $\mathbf{R}$

Bode, Albrecht, Erb, Peter, Hüsing, Helmut Bundestag, Deutscher

Burhenne, Wolfgang, E., Perband, Klaus

Deininger, Rolf

Einführung in die elektronische Datenverarbeitung

Elektronische Datenverarbeitung

Fiedler, Herbert

Giehl, Rudolf
„Analyse zum Datenvolumen und Datenverkehr" in:

Beiträge zur integrierten Datenverarbeitung in der öffentlichen Verwaltung Bayerns, Heft 2. Herausgegeben von der Fa. Siemens AG, Zweigniederlassungen München und Nürnberg; Alfred Weber Institut der Universität Heidelberg

„,Gedanken zur Datenschutzgesetzgebung " in:

ÖVD - Öffentliche Verwaltung und Datenverarbeitung, Heft 0/1971

6. Legislaturperiode.

Stenografischer Bericht über die 101. Sitzung vom 30.9.1970

in: Beiträge zur integrierten Datenverarbeitung in der öffentlichen Verwaltung Bayerns, Heft 1

Herausgegeben von der Fa. Siemens AG, Zweigniederlassungen München und Nürnberg

Die Organisation der betrieblichen Datenverarbeitung, München 1968

Eingabesicherung durch Prüfziffern.

Siemens-Schriftenreihe ,data-praxis"

Datenverarbeitungsstellen - ihre Planung und Organisation. Siemens-Schriftenreihe ,data-praxis“

6. Wahlperiode, Protokoll Nr. 24.

Stenografisches Protokoll über die öffentliche Informationssitzung des Ausschusses für Bildung und Wissenschaft am 3.12.1970.

Tagesordnung: Anhörung von Sachverständigen zur dem Thema: „Wachstumsorientierte Technologien und staatliche Forschungspolitik" betr. den Bereich Elektronische Datenverarbeitung.

EDV-Recht. Berlin 1970.

,Elektronische Datenverarbeitung in der Verwaltung “ in: Staatsanzeiger für Baden-Württemberg Nr. 73/1970

Ein Fernsehkurs im Medienverbund. München 1970.

Eine allgemeine Information.

Herausgegeben von der Firma Siemens AG

,Automatisierung im Recht und juristische Informatik" in: Juristische Schulung, Hefte 9, 11 12/1970, 2, 5/1971

Das Rechenzentrum des Bayerischen Statistischen Landesamtes - 25 Jahre praktische Erfahrung in der maschinellen Datenverarbeitung.

Herausgegeben vom Bayer. Statistischen Landesamt, 1970 
Goppel, Alfons

Grosser, Dietrich

Grundbuchwesen, Elektronische Datenverarbeitung im

Guhr, Lothar

Handbuch der EDV-Organisation Haft, Fritjof

Heilmann, Heidi

Heilmann, Wolfgang,

Reblin, Erhard

Herbold, Rudolf

Herrlitz, Hans

Jaumann, Anton

Informationsmacher, Die

Juristisches Informationssystem

Kerkau, Hans Joachim (A)

,- (B)

Knauber

Langseder, Alois
„Die Bedeutung der Datenverarbeitung für die öffentliche Verwaltung " in: Bayerische Verwaltungsblätter 1971 Nr. 1

„Voraussetzungen für den optimalen Einsatz der EDV“ in: Der Betrieb, Nr. $3 / 1970$

Beiträge zur integrierten Datenverarbeitung in der öffentlichen Verwaltung Bayerns, Heft 3.

Herausgegeben von der Firma Siemens AG, Zweigniederlassung München, 1970.

Planung und Einführung von DV-Verfahren.

Siemens-Schriftenreihe ,data-praxis"

Herausgegeben von IBM Deutschland 1968, zuletzt geändert: Februar 1969

Elektronische Datenverarbeitung im Recht. (EDV und Recht, Band 1). Berlin 1970.

Einsatzplanung für eine Datenverarbeitungsanlage. Stuttgart 1968.

„Datenbanken, Voraussetzungen und Möglichkeiten“ in: Die Computerzeitung, Nr. 11/1971

„Die Datenverarbeitung im kommunalen Bereich" in: Der Bayer. Bürgermeister, Nr. 12/1970

,Automation und Staat" in: data-report Nr. 6/1970

Zur Fragwürdigkeit einer privaten Datenbank für Rechtsdokumentation.

Herausgegeben von der Arbeitsgruppe „EDV und Recht“ im Fachbereich Rechtswissenschaften an der Freien Universität Berlin, 1970

1. Zwischenbericht über die Arbeiten der Projektgruppe. Herausgegeben vom Bundesministerium der Justiz, Bonn, Gesellschaft für Mathematik und Datenverarbeitung, Bonn, C-E-I-R GmbH, Frankfurt.

Bundesanzeiger Nr. 62/1971, Beilage 5

Automatische Datenverarbeitung - Kybernetik in Rechtswissenschaft und Praxis

(Arbeitspapiere Rechtsinformatik Heft 1), Berlin 1970.

„Rechtsfragen der automatischen Datenverarbeitung (ADV) in der öffentlichen Verwaltung " in:

Recht im Amt Nr. 11/1970

„Einsatz der elektronischen Datenverarbeitung im Bereich der Justiz" in:

Justizverwaltungsblatt 1970, S. 25

„Gedanken zum Aufbau eines bayerischen Informationssystems" in:

Bayer. Verwaltungsblätter 1971, Nr. 1 
Löbel, Guido, Müller, Peter, Schmid, Hans

Lübeck, Heinz

Möhl, Kurt

Mühlen, Rainer

A.H. von zur

Müller-Heidelberg jun., Till

Plschek, Hans-Peter

Rohrer, Fritz

Schäfer, Hans

Scheubel, Josef

Schiro, Helmut, Herzog, Reinhart

Schmütsch, Horst

Soziale Probleme der Automation in Bayern Steinmüller, Wilhelm

Thiel, W.

Wenger, $\mathrm{H}$.

Wachtel, Karl
Lexikon der Datenverarbeitung

2. Auflage. München 1969.

Nummerungs- und Schlüsselsysteme bei kooperativer Datenverarbeitung.

AWV-Fachbericht II/III.

Frankfurt 1968.

„Die Datenverarbeitungsfachkräfte bei Eisenbahnverwaltungen" in:

Schienen der Welt, Heft 5/1970.

„Der Computer als Werkzeug und Ziel deliktischer Handlungen im Betrieb" in:

Der Betrieb Nr. 22/1971.

„Rechtsfragen der elektronischen Datenverarbeitung in der öffentlichen Verwaltung " in:

Recht im Amt Nr. 6/1970.

Empfehlungen für die Einplanung eines elektronischen Datenverarbeitungssystems in eine bestehende Betriebsorganisation. Herausgegeben von der Firma Siemens AG

Rechenzentrum, Planung von Bau und Einrichtung.

Herausgegeben von der Firma Siemens AG. Berlin-München 1970.

„Die Funktion der elektronischen Datenverarbeitung in der öffentlichen Verwaltung " in:

Bulletin des Presse- und Informationsamtes der Bundesregierung, Nr. 2/1971

„Stand und Organisation der Datenverarbeitung in der Staatsverwaltung Bayerns" in:

ÖVD - Öffentliche Verwaltung und Datenverarbeitung, Heft $0 / 1971$

Wie sag ich's dem Computer?

München 1970.

„Computer-Mißbrauch - ein kalkulierbares Risiko? “ in:

Der Betrieb, Nr. 22/1971

Herausgegeben vom Bayer. Staatsministerium für Arbeit und soziale Fürsorge, München 1969.

EDV und Recht,

Einführung in die Rechtsinformatik.

Juristische Arbeitsblätter, Sonderheft 6.

Berlin 1970.

On-line,

Datenerfassung und Dialog.

Beiträge zur integrierten Datenverarbeitung in der öffentlichen Verwaltung Bayerns, Heft 4

Herausgegeben von der Firma Siemens AG, Zweigniederlassung München

„Die Datenverarbeitung im kommunalen Bereich“ in:

Der Bayer. Bürgermeister, Heft 12/1970. 
Wanders, Wilhelm

Weyer, Willi

Wolters, Martin F.

Wulf, Harald

Zeitschriften
Nationale und internationale Zusammenarbeit in der Sozialversicherung auf dem Gebiet der elektronischen Datenverarbeitung

Vortrag, gehalten auf dem Bayer. Datenverarbeitungskongreß am 21./22.10.1970 in München

(Auf dem Kongreß zur Verfügung gestelltes Manuskript)

„Die Neuorganisation der elektronischen Datenverarbeitung in Nordrhein-Westfalen" in:

ÖVD - Öffentliche Verwaltung und Datenverarbeitung, Heft 0/71

Der Schlüssel zum Computer.

Düsseldorf und Wien 1969.

Einsatz der Netzplantechnik bei größeren Projekten.

Erfahrungsbericht aus einem Architektur- und Ingenieurbüro. Siemens-Schriftenreihe ,data-praxis“

adl-Nachrichten.

Zeitschriften für Informationsverarbeitung.

Kiel

data-report.

Informationen über Datentechnik. Berlin, München

Die Computer-Zeitung.

Stuttgart

IBM Nachrichten.

Sindelfingen

ÖVD - Öffentliche Vẹrwaltung und Datenverarbeitung.

Stuttgart 